\title{
Comparative Analysis of On-Demand (Pull) Scheduling Algorithm
}

\author{
R. Kavitha \\ Research Scholar, Rayalaseema University, Kurnool, \\ Andhra Pradesh, India
}

\author{
S. Krishna Mohan Rao \\ Gandhi Institute of Technology, Bhubaneshwar, \\ Khurda, India
}

\begin{abstract}
The two most important issues that have to be handled in data dissemination are efficiently exploit the available bandwidth and conserver the energy of mobile devices. In the previous research, Optimal Round Robin (ORR) Scheduling Algorithm for dissemination of varied length data items was proposed. In this paper, another scheduling algorithm which is similar to ORR is proposed and prove that it has better performance when compared in terms of access time. The existing metric stretch is used to propose new algorithm. The analytical model for the proposed algorithm show that it performs well.
\end{abstract}

\section{Keywords}

Access time, bandwidth, broadcast schedule, round robin, waiting time

\section{INTRODUCTION}

Advancement in current technological growth in internet created a high demand for scarce resources in wireless network. It has become a challenge for data scheduling algorithms to use the scarce resources such as bandwidth and power of the mobile devices to be efficiently used. The clientserver model is used in network applications where client sends a request for desired data from the server and the server responses back to the clients. It is assumed that the available bandwidth from server to client is sufficient enough to serve the requests of the clients. In order to serve the clients efficiently and to reduce the access time of their requested data an efficient schedule has to be designed. In this connection there are basically two types of scheduling models: push and pull. According to the authors of $[1,4]$, in push model server proactively disseminates the data items with it based on the precompiled access probabilities of these data items. Pull model discussed in $[3,5,8]$. In this model server doesn't broadcast the data items until unless the clients have put requests. The advantage of both the models is that if one client puts a request it would be served to a large number of clients. In this context [8] has initiated the study of pull/on-demand scheduling. Algorithms of [11] are FCFS, Round Robin, SJF which are studied with respective to operating systems have considered in [6] for scheduling data items with fixed lengths (sizes). $[2,9,10]$ has defined stretch as the metric to consider variable lengths of the data items and defined LWT,MRF, EDF and RXW.

Section 2 presents the model for the proposed algorithm. Section 3 defines the proposed (ERR) algorithm. In Section 4 evaluates experiments on the proposed algorithm. Finally Section 5 concludes the paper with future scope.

\section{MODELING THE PULL (ON- DEMAND) BROADCAST}

This section starts with the designing of a suitable analytical model to evaluate the performance of the proposed pull model. The primary goal of this analysis to is calculate approximately the minimum waiting time for the data items by the clients.

The parameters and the assumptions used in the model are defined as follows:

1. There is a single server which will be serving multiple clients through its downlink channel and receives requests from the clients using uplink. Assume that the capacity of the downlink if greater than the uplink.

2. The database at the server has $\mathrm{N}$ number of distinct data items. i.e. $\mathrm{N}=\{1,2, \ldots, \mathrm{N}\}$

3. Access probabilities represent the popularity of the data items. These items are arranged in sorted order based on their access probabilities $\left\{\mathrm{P}_{1} \geq \mathrm{P}_{2} \geq \mathrm{P}_{3} \ldots \geq\right.$ $\left.\mathrm{P}_{\mathrm{N}}\right\}$. These probabilities follow Zipf distribution [7] with $\theta$ as skew coefficient such that

$$
P_{i}=\frac{(1 / i)^{\theta}}{\sum_{j=1}^{N}(1 / j)^{\theta}}
$$

4. The lengths (sizes) of the data items are randomly distributed. Generally, this size $s_{i}$ (length) is assumed to be the service time of the data items.

5. $\lambda$, is the arrival rate of the pull system.

6. The metric stretch is defined as $S(i)=\sum_{k \epsilon Q_{i}} \frac{1}{s_{i}}$ where $Q_{i}$ is the request queue of the data item $i$

\section{1) Minimum Expected Waiting Time:}

As the primary goal of the proposed algorithm is to minimize the average access time of the data items by the clients, this indirectly means that the waiting time has to be reduced. In this algorithm the waiting time of data items is calculated, which is similar to the traditional round robin technique. Every item has to be assigned with time quantum till it is broadcasted completely. Waiting time is calculated as the difference between the total times the item has waited with the actual service time $\left(\mathrm{wt}_{\mathrm{i}}\right)$. Then calculate average waiting time as

$$
\operatorname{avgwt}=\sum_{i=1}^{N} w t_{i} \times P_{i} \times \lambda \ldots E q-1
$$

Where $P_{i}$ is the access probability of data item $i$ and $\lambda$ is arrival rate. Then average access time is calculated as

$$
\text { Avg }_{\text {accesstime }}=(1 / b) \times(\operatorname{avgwt} / N) \ldots E q-2
$$




\section{EFFICIENT ROUND ROBIN SCHEDULING ALGORITHM}

\section{Procedure ERR Algorithm}

Input : $\mathrm{N}$ data items

Output: Average Access Time

Begin

1. Randomly generate the sizes of the data items

2. Calculate the stretch of each item

3. Arrange the data items in sorted order based on stretch values (from highest to lowest)

4. Repeat steps 5 through for all data items

5. If $\left(\left(\mathrm{s}_{\mathrm{i}}\right)>\operatorname{tq}\right.$ and $\left.\left(\mathrm{s}_{\mathrm{i}}-\mathrm{tq} \leq \mathrm{tq}\right)\right)$

Broadcast the entire data item

Else Broadcast a part of it for a period of time quantum

6. Calculate waiting time of all the data items

7. Calculate average waiting time using Eq-1

8. Calculate average access time using Eq-2

\section{Figure 1 : ERR Algorithm}

\subsection{An Example:}

Consider the Table 1 which shows the data items with respective service times and access probabilities. Using the data given in Table 1 calculate stretch for each data item.

The Stretch is calculated as $P_{i} / s_{i}$.

Table 2 illustrates the calculated stretch values. After calculating stretch for each data item, arrange the data items in the descending order with respect to stretch values.

Table 3 gives the ordering of the data items based on stretch values.

Consider the time quantum as 4 , tq $=4$. Based on the algorithm of [12] and the proposed algorithm calculate the waiting time of each item using time quantum from Table 3 .

Table 1. Show data items with service times and access probabilities

\begin{tabular}{|c|c|c|}
\hline Data Item $\mathbf{D}_{\mathbf{i}}$ & Service Time $\mathbf{s}_{\mathbf{i}}$ & Access Probabilities $\mathbf{P}_{\mathbf{i}}$ \\
\hline D1 & 10 & 0.0309 \\
\hline D2 & 5 & 0.21 \\
\hline D3 & 5 & 0.17 \\
\hline D4 & 8 & 0.1547 \\
\hline D5 & 6 & 0.1383 \\
\hline
\end{tabular}

Table 2. Shows calculated Stretch values of each data item

\begin{tabular}{|c|c|c|}
\hline Data Item $\mathbf{D}_{\mathbf{i}}$ & Service Time $\mathbf{s}_{\mathbf{i}}$ & Stretch S(i) \\
\hline D1 & 10 & 0.0309 \\
\hline D2 & 5 & 0.043 \\
\hline D3 & 5 & 0.035 \\
\hline D4 & 8 & 0.0193 \\
\hline D5 & 6 & 0.023 \\
\hline
\end{tabular}

Table 3. Sorted data items based on stretch values

\begin{tabular}{|c|c|l|}
\hline Data Item $\left(\mathbf{D}_{\mathbf{i}}\right)$ & Service Time $\left(\mathbf{s}_{\mathbf{i}}\right)$ & Stretch S(i) \\
\hline D2 & 5 & 0.043 \\
\hline D3 & 5 & 0.035 \\
\hline D1 & 10 & 0.0309 \\
\hline D5 & 6 & 0.023 \\
\hline D4 & 8 & 0.0193 \\
\hline
\end{tabular}

Algorithm ORR [12] has two phases, where in first phase all the data items are allocated for a time period of time quantum using table 3 and the remaining amount of service time are calculated.

They are then broadcasted in ascending order of their service time in the second phase. The allocation of data items for broadcast is :

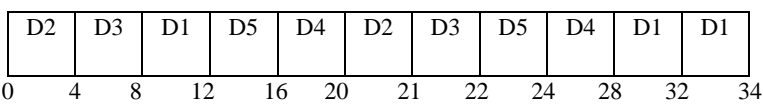

Calculate the waiting time of the data items

Table 4. Waiting Time of each data item

\begin{tabular}{|c|c|}
\hline Data Item $\left(\mathbf{D}_{\mathbf{i}}\right)$ & Waiting Time $\mathbf{~ w t ~}_{\mathbf{i}} \mathbf{)}$ \\
\hline D2 & $21-5=16$ \\
\hline D3 & $22-5=17$ \\
\hline D1 & $34-10=24$ \\
\hline D5 & $24-6=18$ \\
\hline D4 & $28-8=20$ \\
\hline
\end{tabular}

Now calculate average waiting time of the data items using Eq1 and Average Access time of the data items is calculated as Eq2 .Therefore the average access time for the above example is 7.8199 broadcast units.

According to Proposed ERR algorithm data items are broadcasted as : 


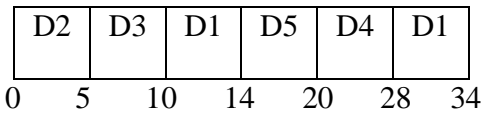

Waiting times of the data items are shown in Table 5.

Average waiting time and average access time of the data items are calculated using Eq-1 and Eq-2. And Average access time for the given data is 5.340 broadcast units.

It is observed that the average access time based on ORR algorithm is 7.8199 where as proposed ERR has 5.340.

Hence from the example it is proved that the proposed algorithm performs better in terms of access time.

Table 5. Waiting Time of data items w.r.t. ERR algorithm

\begin{tabular}{|c|l|}
\hline Data Item $\left(\mathbf{D}_{\mathbf{i}}\right)$ & Waiting Time $\left(\mathbf{w} \mathbf{t}_{\mathbf{i}}\right)$ \\
\hline D2 & $5-5=0$ \\
\hline D3 & $10-5=5$ \\
\hline D1 & $34-10=24$ \\
\hline D5 & $20-6=14$ \\
\hline D4 & $28-8=20$ \\
\hline
\end{tabular}

\section{EXPERIMENTAL RESULTS}

In this section proposed on-demand (pull) system is validated by performing simulation experiments. The main goal of the system is to reduce access time of the data items.

The assumptions and parameters which are used for the simulation are as follows:

1. Simulation experiments are evaluated with a single server containing number of data items $(\mathrm{N})$ varying from $100-500$

2. The arrival rate, $\lambda$, of the pull system is assumed to vary between 5-25.

3. Sizes of the data items generated between from 110.

4. The access probabilities of the data items to be skewed, change $\theta$ from $0.2-0.8$

5. Time quantum, tq, is set to vary from 4-7.

6. Bandwidth, $b$, of the channel varies from 10-50

7. To compare the performance ORR algorithm of [12] is chosen as point of reference.

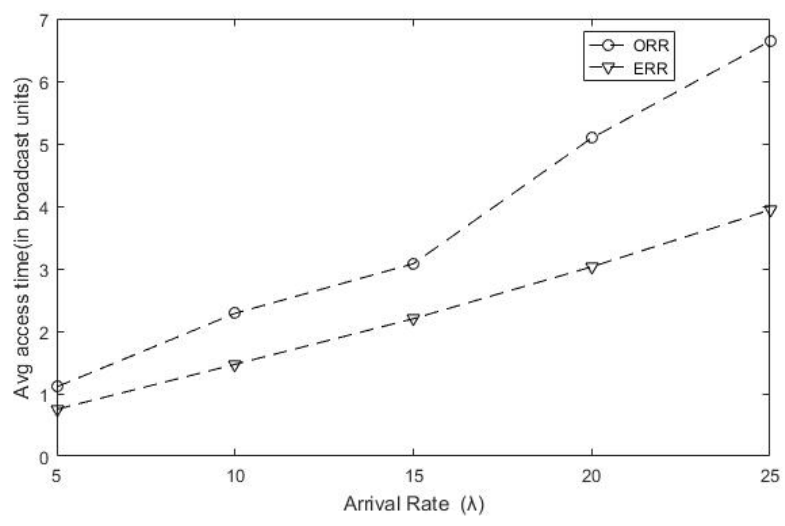

Figure 1: Impact of Arrival rate on Average Access Time

From Figure 1 it is concluded that as the value of arrival rate, $\lambda$, increases the average access time also increases but at moderate rate when compared with [12].

Figure 2 shows the variation of average access time with different values of bandwidth (b). It is observed that the access time drastically falls as there is increase in the bandwidth which is the capacity of the broadcast channel.

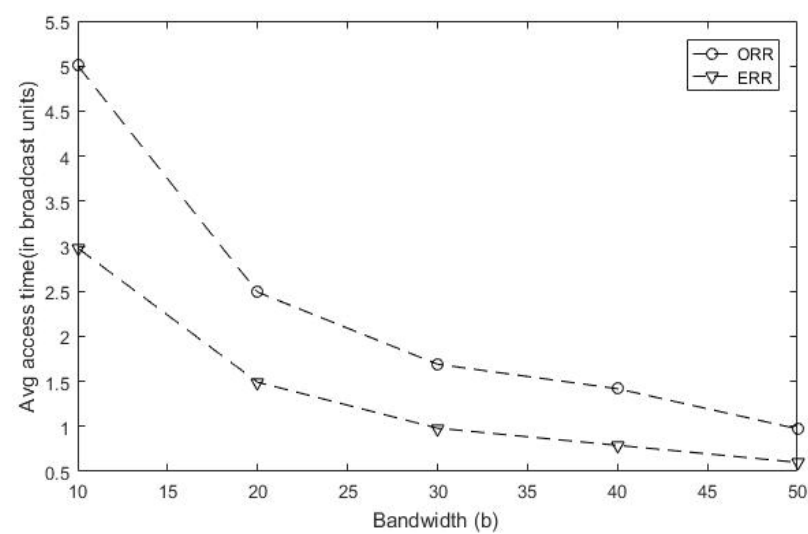

Figure 2: Performance comparison with varying Bandwidth

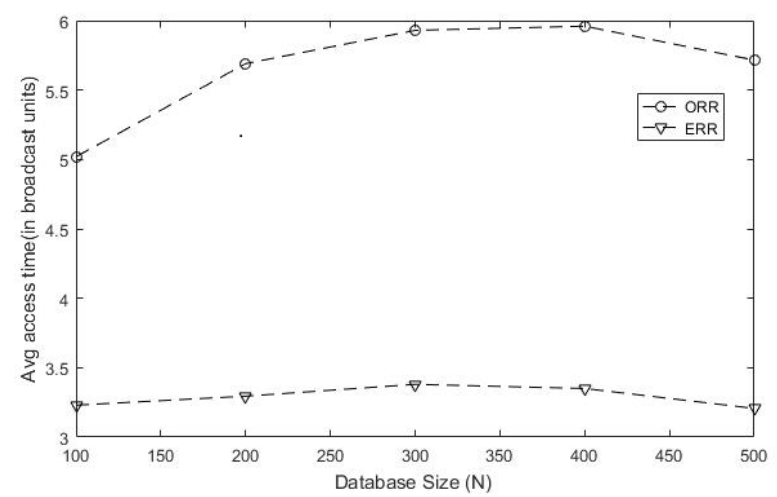

Figure 3: Performance comparison with varying Database Size

Figure 3 demonstrates that as the size of the database or number of data items increases the access time also increases. And ERR performs better than that of ORR. 


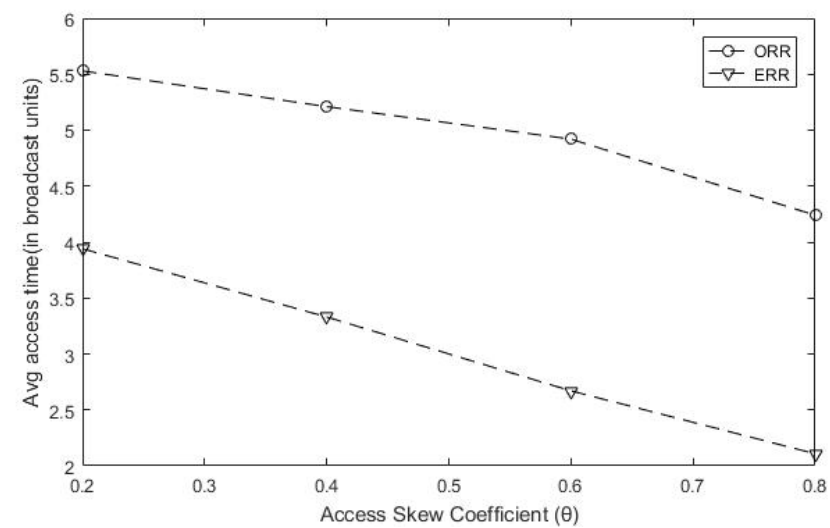

Figure 4: Performance Comparison with Varying Access Skew Coefficient

Figure 4 shows that as the access skew coefficient increases there is a decrease in the access time. Again it is observed that there is a significant variation of average access time.

Figure 5 demonstrates how access time varies with the most important parameter of the algorithm, the time quantum. As the time quantum increase there is a decrease in average access time.

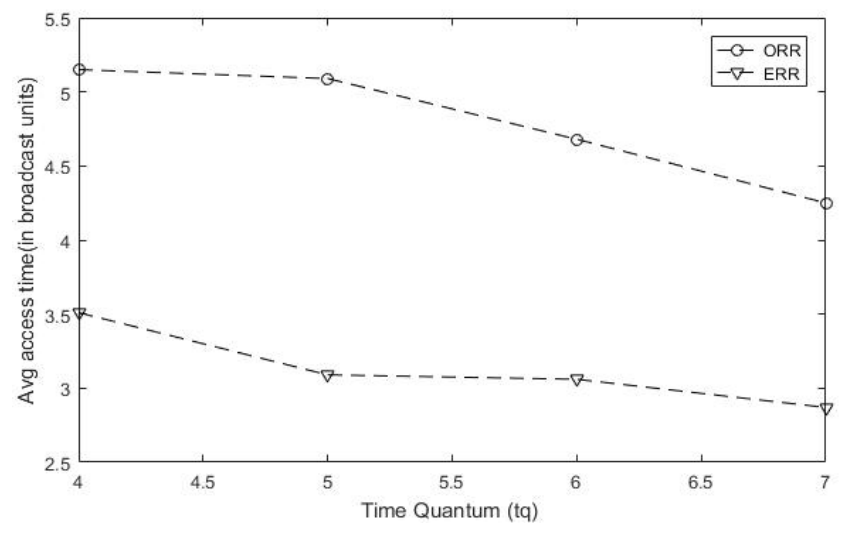

Figure 5 : Average Access Time vs. Varying Time Quantum

Figure 6 demonstrates the change in average access time with varying database size (100 - 500) and for time quantum (6 and 8).From the analysis it is concluded that the proposed algorithm outperforms in terms of average access time with varying arrival rate, bandwidth, database size, time quantum and access skew coefficient.

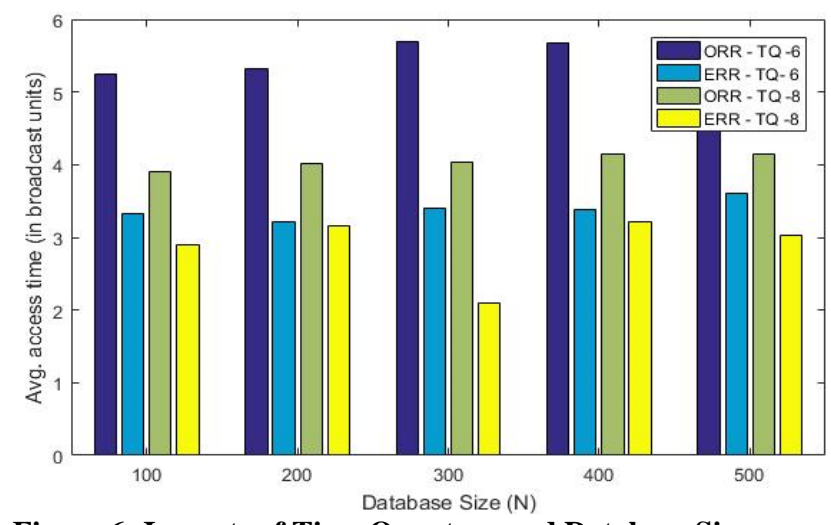

Figure 6: Impacts of Time Quantum and Database Size on Average Access Time

\section{CONCLUSION}

This paper has developed broadcast program for pull system given the access probabilities and sizes (lengths) of the data items. ERR algorithm is developed to minimize the access time in pull model. Sensitive analysis is conducted on ERR algorithm to measure its performance on parameters such as database size, access skew coefficient, time quantum, bandwidth and arrival rate. It is proven by the above results that the proposed algorithm performs better for variable length data items. The future work can be extended to develop a hybrid model to reduce access time for disseminating the data items on to multiple channels.

\section{ACKNOWLEDGMENTS}

Our Sincere thanks to the experts, authors and publishers listed in the reference as without their contribution this paper wouldn't have completed.

\section{REFERENCES}

[1] S. Acharya, R. Alonso, M. Franklin, and S. Zdonik. Broadcast disks: Data management for asymmetric communications environments. In Proceedings of ACM SIGMOD Conference on Management of Data, pages 199-210, San Jose,CA, USA, May 1995

[2] D. Aksoy and M. Franklin. R x W: A scheduling approach for large scale on demand data broadcast.IEEE/ACM Transactions on Networking, 7(6):846-860, December 1999.

[3] S. Acharya and S. Muthukrishnan. Scheduling on demand broadcasts: New metrics and algorithms. October 1998.

[4] S. Hameed and N. H. Vaidya. Efficient algorithms for scheduling data broadcast In Wireless Networks, Vol. 5, pages 183-193, 1999.

[5] H. D. Dykeman, M. Ammar, and J. W. Wong. Scheduling algorithms for videotext systems under broadcast delivery. In Proceedings of IEEE International Conference on Communications (ICC'86), pages 1847 1851, Toronto, Canada, June 1986.

[6] J. W. Wong. Broadcast delivery. Proceedings of the IEEE, 76(12):1566-1577, December 1988

[7] G. K. Zipf. Human Behaviour and the Principle of Least Effort. Addison Wesley, MA, USA, 1949

[8] D. Aksoy and M. Franklin. R x W: A scheduling approach for largescaleondemand data broadcast. IEEE/ACM Transactions on Networking, 7(6):846-860, December 1999

[9] Q. L. Hu, D. L. Lee, and W.C. Lee. Performance evaluation of a wireless hierarchical data dissemination system. In Proceedings of the 5th Annual ACM/IEEE International Conference on Mobile Computing and Networking (MobiCom'99), pages 163-173, Seattle, WA, USA, August 1999.

[10] Y. Wu and G. Cao. Stretch-Optimal Scheduling for OnDemand Data Broadcasts. IEEE International Conference on Computer Communications and Networks, pages 500.504, Oct. 2005.

[11] A. Silberschatz and P. Galvin. Operating System Concepts. Addison Wesley, 1997. 
[12] R. Kavitha, S.Krishna Mohan Rao “,Optimal Round Robin (ORR) Scheduling for On-Demand Broadcast in Mobile Computing Environment", IJARCSSE , vol 3, sep-2013, pp-1014-1019

\section{AUTHOR'S PROFILES}

R. Kavitha, research scholar in Computer Science bearing registration no PP.COMP.SCI-268, from Rayalaseema
University, Andhra Pradesh,India. Her area of interest is Design Analysis and Algorithms, Mobile Computing, Database Management System.

Dr. S .Krishnamohan Rao, Principal, Gandhi Institute of Technology, Bhubaneshwar, Khurda, India. His areas of specializations are Mobile Computing, MANETS, Adhoc Sensor Networks and Computer Networks. 